\title{
Prevalence of Oral Habits in Dentalanomalies
}

\author{
Rozela Xhemnica \\ $\mathrm{PhD}$, University of Medicine of TiranaFaculty of Dental Medicine \\ Elsena Mataj \\ University of Medicine of TiranaFaculty of Dental Medicine \\ Milton Rroço \\ MD. Msc.University of Medicine of TiranaFaculty of Dental Medicine
}

\section{Abstract}

There are a lot of oral habits in which are included even non-nutritional habits. The role of these habits has been evidenced in the development of different malocclusion in each plane, vertical, transversal and sagittal ones. The reason why habits can cause such problems is based on equilibrium theory. The main purpose of this study is to assess the prevalence of nonnutritional habits and their effect development of dental anomalies. The method of this study was descriptive in prospectively. Its duration was 20 months. The age of children taken in this study vary from 6 to 15 years old and was selected casually at clinics of "University of Mother of Good Council" and two private clinics. Results we were able to fill the questionnaires of 194 patients in which $50.3 \%$ of them were females and $43.1 \%$ of them were males. According to the age of children, there was a frequency of $46.7 \%$ of $6-10$ years old children and $53.3 \%$ of 11-15 years old ones. Results on the frequency of non-nutritive habits were about $64 \%$ of fake pacifier usage, in which there was a variety of percentage of time when it was used. Meanwhile, thumb sucking resulted in $10.20 \%$ of results, lip biting or sucking $7.10 \%$. Conclusions: This group age was very often in predisposition to have such habits. Our results confirm the impact of these habits in the development of different malocclusions. We recommend applying preventive strategies so we can avoid the installation of these habits and such malocclusions.

Keywords: malocclusion, oral habits, developing, preventive, dental anomalies.

\section{Introduction}

There are a lot of oral habits, where in this article we will focus at non-nutritive oral habits. The habit is as the visible part of an iceberg, while the consequence, the major part, is under water. The consequences of this habit consist of the manifestation of malocclusion. Malocclusion is defined as abnormal positions of teeth or a non correct proportion of jaws. (1) These malocclusions can be classified according to the 
transversal, sagittal and facilitated vertical plan as: Transversal (median line mismatch, scissors bite, cross bite posterior, edge to edge bite); Sagital (protrusion, retrusion, II molar class and III molar class); Vertical (open bite, deep bite, edge to edge bite, teeth supra and infra occlusion). The manifestation of this malocclusion from the implication of oral habit is explained by the equilibrium theory. This theory elucidates that if equal force is exerted on an object then it will stay in the same position, thus the forces are in equilibrium. The opposite will happen if unequal forces are exerted on an object, it will move in the direction of the greatest force exerted.(2). In oral cavity there is a harmonic balance between the pressure of cheeks, tongue and lips which will lead to a normal dento-facial and skeletal growth. In that moment when a habit interferes, this balance will disrupt and we will have an abnormal pressure of soft tissue, alternation of the muscles. This will lead to altered dento-facial and skeletal growth. (3) The purpose of this study was to evaluate the prevalence of non nutritional suctioning habits and their impact in the development of dental abnormalities. Objectives consisted of estimating the number of cases with non nutritional suctioning habits, orthodontic evaluation of malocclusions caused by the habits, estimating the distribution of these habits by sex and age, and evaluating possible linkages of casual factors and development of oral abnormalities.

\section{Methodology}

This study was a descriptive prospective one that lasted for a period of 20 months (November 2017- June 2019). Children of the age group of 6-15 years were randomly presented in dental clinics. In total, data on 194 individuals were collected. The inclusion criteria of these individuals consisted of: The presence of deciduous canine and first molar; the presence of permanent canine and first molar; all previous orthodontic treatment services and / or more; no cranio-facial abnormalities (different cleft or syndromes). These data were collected in clinics like the stated one in the Faculty of Dental Medicine, in the University of "University of Mother of Good Council" and in two private dental offices by writing down a standard form (4) This form was divided into a consensus section and three sections (general information, anamnesis and clinical extraoral and intraoral examination) to be completed by the physician. Time for the examination was approximately 20 minutes and we did take care for the control of infection. To minimize mistakes was chosen specialist physician with knowledge in this field. 


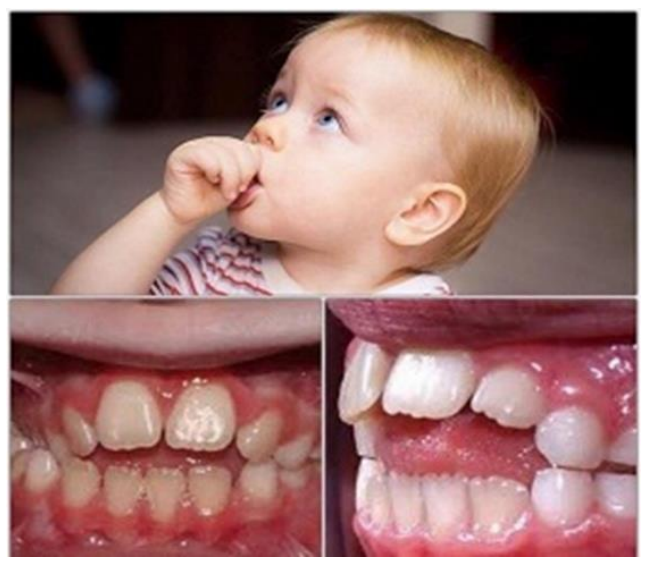

\subsection{Statistical analyses}

Data were processed in statistical software such as SPSS 19.00 and Excel 13.00. The links that were reached were considered significant if the p-value $\leq 0.05$ and proved by testHi-square.The results were presented in tables and simple graphics and were also compared with articles with the same focus, found in PubMed and Cochrane.

\section{Results}

In this study we found that we had $43.10 \%$ males and $50.3 \%$ females while the frequencies of the age group were distributed in $46.7 \%$ for $6-10$ years old and $53.3 \%$ for 11-15 year old. Based on the results $64 \%$ had used pacifier respectively $16.8 \%$ only during sleep, $21.8 \%$ only when they cried and $23.8 \%$ every moment. It was found a relation between using pacifier and the molar class in both right and left side with p-value 0.03 and 0.04. (Graphic.1,2).

Graphic.1:

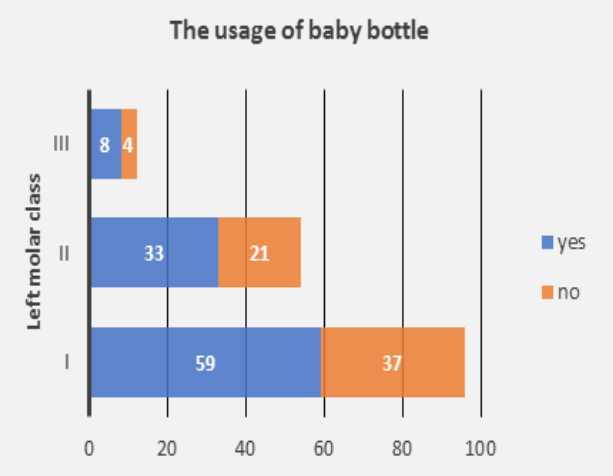

\section{Graphic.2:}

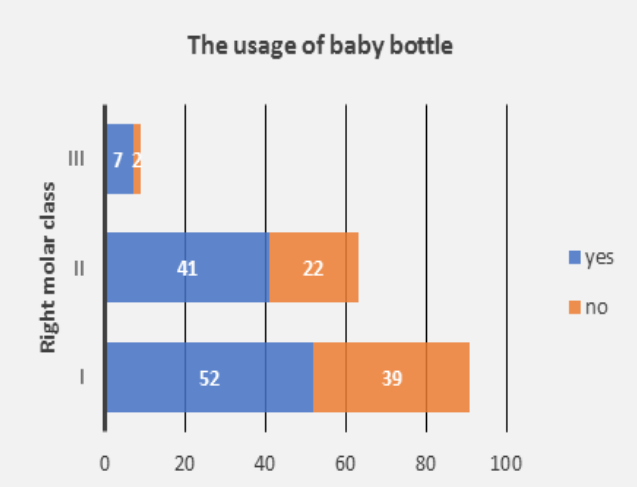

The other malocclusions that can relate with the pacifier as a cause of their presence are over-jet (graphic. 4), cross-bite(graphic .3) and dental crowding (graphic .5).We can say that it came to a very significant connection especially with the cross bite 
where p-value resulted 0.000 . (graphic.3) there was also a strong connection between this type of malocclusion and pacifier usage.

Graphic.3

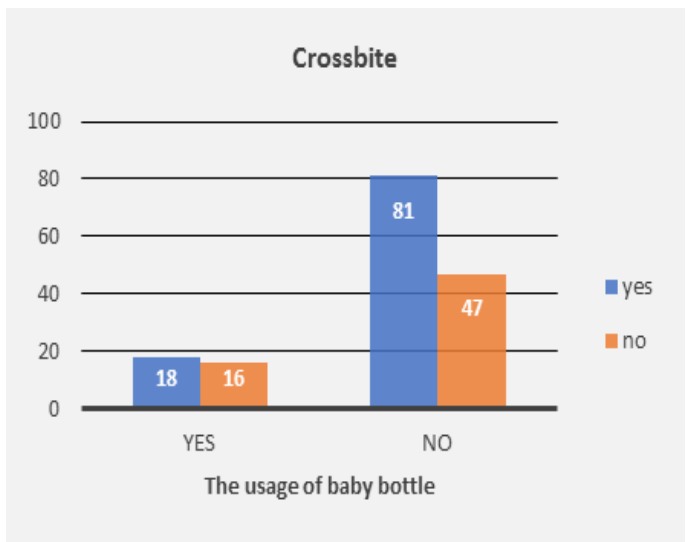

Graphic. 4

The usage of baby bottle

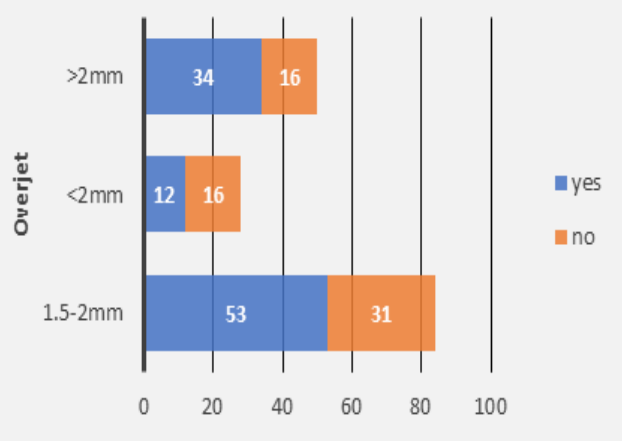

\section{Graphic.5}

The usage of baby bottle

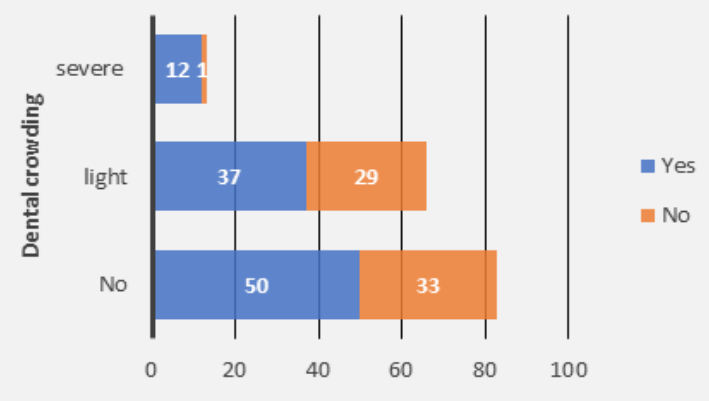


Other habits like thumb suctioning or the suctioning / biting of the lips resulted in frequencies correspondingly $10.20 \%$ and $7.10 \%$. Most predisposed to these addictions were children of the age group 11-15 years old, while there were no statistically significant differences regarding gender. These other non nutritional suctioning habits resulted to have significant statistically relation with right and left molar class, canine class and open bite.

\section{Discussion}

In our study as we emphasized before non nutritional suctioning habit resulted in frequencies 58\%. Other authors referred frequencies from $25.5 \%$ (5) up to $88 \%$ (6). This sensitive difference can happen for many reasons. First, in our study these habits are considered present only if the clinical examinations and the information that parents referred were in correlation, which is not specified in other studies whether it has been evaluated in this way or not. Second it varies because of the desire of parents to participate in these relevant studies. It is important to emphasize the difference between the results of our study and the results of Quashie-Williams, which is as the result of the different age group considered, respectively 6-15 years old and 11-13 years old. Also our result on the absence of any significant difference between habit and gender was supported by other authors. (7) While age-related results varied slightly differently in Shetty \& Munshi's studies referring to higher frequencies in the 12-year-old age group, which would decrease with increasing age.(8)The relation between these habit and molar class and canine class were supported also from other authors like Lux et al, (9) and P. Cozza (10) who had done similar studies respectively in Germany in the age group of 9 years and in Albania in the age group of 7-15 years. Another anomaly with which a statistically significant association was obtained for non nutritional suctioning habit was open bite as we mentioned before 9.94\% of individuals had at least one non nutritional suctioning habit and open bite, a result different from that of Martin et al. which reported prevalence of 38.5\%. (11) This difference may be due to the disregard of the duration of the disruption in different children and the self-corrections that may have occurred from early disruption of the habit. Cross bite is one of the most frequently analyzed malocclusions due to the use of pacifier. This fact was also confirmed by us, which resulted in a frequency $11.11 \%$ of babies using pacifiers to have cross bites with a very strong significative relation ( $p$-value $=0.000$ ) confirmed by many other authors where among them Heimer et al referenced a prevalence of 10.04\%.(12) However, there were other confirmations by other authors of higher prevalence such as Gomes \& Silva et al. with a prevalence $29.05 \%$.(13) This difference may be due to the variation in timing of the use of pacifier or the combination of its use and the breast feeding in different individuals.

\section{Conclusion}

Based on the goals we set, in the entirety of the prevalence, in this investigation the prevalence of non nutritional suctioning habit resulted in high percentage almost 
$58 \%$ and more expressed in 11-15age group. Knowing the etiology of malocclusion is essential to the success of an orthodontic treatment because the prerequisite for correction is to eliminate the cause. From this investigation we concluded and reinforced the opinions of various authors on the association of non-nutritious suctioning habit with dental abnormalities as follows: The connection between these habits and second molar (38.9\%) and canine (38.9\%) class was evident; individuals that have at least one of these non nutritional suctioning habit have the possibility to have the reduction of vertical height, thus predisposition for open bite; in our study $11.11 \%$ of children who had used the pacifier had open bite; the link between pacifier use and dental crowding was evident where 37 and 12 children (out of 162) had light and heavy crowding, respectively.

\section{Recommendations}

These data can provide the basis for planning preventive strategies to eradicate oral habits and reduce the chance of malocclusions,furthermore contributing to an increase in the national level of oral health.Dentists, along with other health professionals, should educate parents.The latter should make sure that there is no need to worry if their child has an addiction to school age,time to try to encourage the child to stop the habit in order to reduce the potential harmful effects on the occlusion. However, we believe that these kinds of problems require close cooperation between the various specialists (pediatrician, allergist, orthodontist and speech therapist) and early orthodontic visit and treatment, when needed in children with bad habits, allergic rhinitis and / or adeno-tonsillar hypertrophy. This will allow for early detection and timely treatment of dysfunctions to avoid the deterioration of already occurring malocclusions. The method to reduce the number of individuals with habits may include the use of provisional devices that reduce the active child's habits and subsequently the use of devices to correct malocclusions.

\section{References}

[1] Dewanto H. Aspek-aspekepidemiologimaloklusi. Yogyakarta: Gajahmada University Press.2004

[2] Davidovitch M, Rebellato J: Utility arches: a two-couple intrusion system, Sem Orthod 1:25-30,1995

[3] Davidovitch M, Rebellato J: Utility arches: a two-couple intrusion system, Sem Orthod 1:25-30,1995

[4] Pereira et al. Thayse Steffen; Association between harmful oral habits and the structures and functions of the stomatognathic system: perception of parents/guardians. CoDAS 2017;29(3):e20150301 DOI: 10.1590/23171782/20172015301.2017

[5] Kharbanda OP, Sidhu SS, Sundaram KR, Shukla DK. Oral habits in school going children of Delhi: a prevalence study. J indian soc pedoprev dent september 21 (3): 120-24.2003. 
[6] Sidlauskas A, Lopatiene K: The prevalence of malocclusion among 7-15-yearold Lithuanian schoolchildren. Medicina (Kaunas); 45: 147-52.2009.

[7] Quashie-Williams R, Dacosta 00, Isiekwe MC. The prevalence of oral habits among 4 to 15 year old school children in Lagos, Nigerian Journal of Health and Biomedical Science;6 (1): 78-82.2007.

[8] Shetty SR, Munshi AK. Oral habits in children: aprevalence study. J ind soc of pedoprev dent jun; 17(2):61 - 6.1998.

[9] Lux CJ, Ducker B, Pritsch M, Komposch G, Niekusch U. Occlusal status and prevalence of occlusal malocclusion traits among 9-year-old schoolchildren. Eur J Orthod; 31:294-9.2009.

[10] Giuseppina Laganà1, Caterina Masucci2*, Francesco Fabi3, Patrizio Bollero1 and Paola Cozza1. Prevalence of malocclusions, oral habits and orthodontic treatment need in a 7- to 15-year-old schoolchildren population in Tirana Progress in Orthodontics, 14:12.2013.

[11] Martin et al, Warren J, Slayton L, Yonesu T et al: Effects of nonnutritive sucking habits on occlusal characteristics in the mixed dentition. Pediatr Dent; 27: 445-50.2005.

[12] Heimer et al.,Karjalainen S, Ronning O, Lapinleimu H, et al. Association between early weaning, non nutritive sucking habits and occlusal anomalies in 3-year-old Finnish children. Int J Paediatr Dent ;9:169-73.1999.

[13] Goems\& Silva, Ovsenik M. Incorrect orofacial functions until 5 years of age and their association with posterior crossbite. Am J Orthod Dentofacial Orthop ;136:375-81.2009. 